\title{
5 Research Square

\section{Sagittal imbalance of the spine is associated with poor sitting posture among primary and secondary school students in China: A cross-sectional study}

\section{Li Chaoqun}

Nanjing Sport Institute

Zhao Yuqi

Tianjin University of Sport

Yu Zhenghui

Tianjin University of Sport

Han Xu

Tianjin University of Sport

Wen Li ( $\nabla$ wenli34@hotmail.com )

Nanjing Sport Institute

\section{Lin Xiang}

New Jersey Institute of Technology

\section{Research Article}

Keywords: Children, Posture, Spine, Sagittal angle of the spine, Spinal anomalies

Posted Date: June 25th, 2021

DOI: https://doi.org/10.21203/rs.3.rs-558657/v1

License: (1) This work is licensed under a Creative Commons Attribution 4.0 International License. Read Full License 


\section{Abstract \\ Background}

There's no definite answer as to how writing posture affects students' spine. This study attempted to compare the sagittal curvature of the spine between sitting and standing postures in adolescents. To reveal the variation rule of spinal sagittal curvature of students with learning posture, and to discover the key factors that may affect students' spinal health.

\section{Methods}

1138 participants (male, 604; female, 534; age range, 6-18 y) from three schools in Tianjin, China, including 570 primary school students and 568 secondary school students. This study used SpineScan and PA200 Station Posture Assessment System to assess the sagittal curvature of the spine for three postures: sitting on a chair in an upright position, seated at a desk while reading/writing, and standing in a natural relaxed position. Analyze the difference of the spine angle of the three postures and the correlation between the sagittal plane angle of the spine and body posture.

\section{Results}

When some teenagers sat reading/writing, the sagittal angle of the spine markedly changed, with the lumbar lordosis angle significantly decreased $(p<0.05)$ and the thoracic kyphosis angle significantly increased $(p<0.05)$. These angles interact with each other and were positively correlated with the height of the teenager $\left(R^{2}=0.179 ; p<0.05\right)$. By contrast, teenagers with lumbar lordosis and thoracic kyphosis angles within standard references ranges in a seated reading/writing posture also had good spine shape while standing.

\section{Conclusions}

Compared with standing posture, the angle of thoracic kyphosis gradually increases from upright sitting to reading/writing., Lumbar lordosis significantly decreasing or even disappearing, and the flexion of whole spine will increase the risk of spinal injury. Height is an important factor affecting the shape of students' sitting spine.

\section{Background}

The spine constitutes the central axis and pillar of the human torso, and its four physiological bends in the sagittal plane are a biomechanical masterpiece $[1,2]$. In addition to the shape of the cone and intervertebral disc of the spine itself, the muscles acting on the spine related to the maintenance of posture also play an important role in the physiological bends of the spine. Unbalanced contraction of 
muscles antagonizing each other in function will lead to changes in the physiological curvature of the spine [3]. The physiological curvature of the spine is closest to the natural state that reduces energy consumption and maintains the curvature in the most relaxed state in a natural standing position. By contrast, in a sitting position, the balance of the spinal curvature is pulled by the muscles and deviates from this relaxed state, resulting in excessive kyphosis of the thoracic vertebrae and a reduction or disappearance of lumbar lordosis [4, 5]. Existing studies have shown that sitting for a long period greatly changes the tension of the body's soft tissues [6]. Prolonged poor sitting posture over time is a root cause of spinal abnormalities. This is exacerbated among people, for example students, who use desks and chairs unsuited to their height $[7,8]$.

The change in body posture that deviates from the normal state is called an abnormal posture and is one of the most serious problems related to normal physical development in children $[9,10]$. The skeletal systems of young school-age children are in the developmental stage, and their spines are characterized by rapid growth and high susceptibility to diseases caused by external factors [11,12]. During this period, the stabilization of the anterior and posterior curvatures of the spine has not been completed. When children begin attending school, their lifestyle is markedly changed, as they are required to spend more time seated at desks and chairs then they had before attending school. Long-term incorrect posture beginning at this stage may affect the morphological development of the spine [13].

Few studies have examined the effect of poor reading/writing posture on the sagittal shape of the spine in children and teenagers or investigated the differences in the sagittal shape of the spine among teenagers associated with different sitting positions. To address these issues, we tested the hypotheses that (1) the sagittal curvature of the spine exceeds the normal range in students with poor posture, (2) that this abnormal curvature range is associated with spinal sagittal imbalance, (3) and that this association is decreased when proper sitting posture is maintained.

\section{Methods}

\section{Participants}

In total, 1138 students (male, 604; female, 534; age range, 6-18 y) from three schools in Tianjin, including 570 primary school students (the enrollment age for primary school in China is 6 years old, and children typically attend primary school for a total of 6 years) and 568 secondary school students (age range, 11-18; a total of 3 years in secondary school), were enrolled in the study.

\section{Study design}

The thoracic spine kyphotic angle (TKA) and the lumbar lordosis angle (LLA) of all participants were measured with the SpineScan hand-held spine-measuring device (OrthoScan) while participants maintained a natural relaxed standing position or an upright sitting position or were seated at a desk reading/writing. The SpineScan device has shown good measurement reliability in determining TKA and 
LLA [14], and it is more efficient and suitable than using an imaging measurement method for a study with a large sample size.

During the measurements, the students were asked to stand naturally and then to sit on the chairs used in their classes. The students were asked to straighten their backs, and the curvature for the upright sitting position was immediately measured. They were then asked to remain seated at their desk and to copy a paragraph of text. Measurements of the spinal curvature at the desk during reading/writing were obtained after $5 \mathrm{~min}$. Each posture was measured three times, and the averages of each of the three results were calculated.

The measurements of the desks and chairs used by the students in this study followed the GB/T39762014 Standard 38 of Functional Dimensions and Technical Requirements of School Table and Chair proposed by the General Administration of Quality Supervision, Inspection and Quarantine of China [34]. Thus, primary and secondary school students have different sized desks and chairs. The heights of desks and chairs used by secondary school students (grades 7-9) are $700( \pm 2) \mathrm{mm}$ and $400( \pm 2) \mathrm{mm}$, respectively, whereas those used by primary school students (grades 1-6) are $610( \pm 2) \mathrm{mm}$ and $340( \pm$ 2) $\mathrm{mm}$, respectively.

The height and weight of each participant were measured with a standard ruler and weight scale. The vital capacity of the participants was measured using the FGC-A + Lung Function Instrument. They were asked to breathe in as much air as they could and then to forcefully exhale through the blower as hard as they could, blowing for three consecutive 15 -s intervals.

The pelvic inclination and neck inclination of participants' standing postures were measured using a PA200 Station Posture Assessment System.

We randomly selected 50 participants, and two investigators repeatedly measured the participants spinal angles. Cronbach's alpha coefficient for the two experimenters was 0.96 , indicating a high reliability of the measurements [15].

Data were analyzed using SPSS, version 25 (SPSS Inc.). One-way ANOVA were used to analyze the sagittal angles of the spine for the three postures. Independent-samples $t$ test analyses were used to assess vital capacity and thoracic curvature. Two-side $p$ values $<0.05$ were considered statistically significant. The Pearson coefficient was used to test the correlation between variables, and regression analysis was used to determine their linear relationship.

\section{Results}

The average TKA value measured in 1138 participants was smallest in the upright position, increased when standing naturally, and reached a maximum when participants were seated and reading/writing at their desks. The average LLA value was smallest in the natural standing position but was absent in many 
participants when they were reading/writing at their desks, resulting in lumbar kyphosis. Significant differences were observed in TKA and in LLA for the three different postures (Table 1).

Table 1

Average thoracic kyphosis angles (TKAs) and lumbar lordosis angles (LLAs) for three postures in all participants.

\begin{tabular}{|llll|}
\hline Angle Measured & \multicolumn{2}{l}{ Body Posture } \\
\cline { 2 - 4 } & Natural Standing & Upright Sitting & Seated Reading/Writing \\
\hline TKA & $32.873(8.669)^{\#}$ & $25.138(8.729)^{*}$ & $33.529(9.706)^{\#}$ \\
\hline LLA & $-26.043(10.615)^{\#}$ & $-12.661(9.063)^{*}$ & $0.772(12.676)^{* \#}$ \\
\hline Numerical values represent the mean (standard deviation). & \\
\hline \multirow{*}{*}{$\mathrm{p}<0.05$ compared with natural standing position; \#p < 0.05 compared with upright sitting position. } \\
\hline
\end{tabular}

Participants were grouped according to their age, one group for each age from 6 to 18 years old (13 groups), and the mean values of TKA and LLA were calculated for each age group, by sex (Table 2). These descriptive data appeared to show that both thoracic kyphosis and lumbar lordosis in male and female participants tended to increase with age. The TKA in male participants was generally greater than that of females of the same age, and participants' thoracic curves seemed to have a small growth spurt at age 11 . 
Table 2

Mean thoracic kyphosis angles (TKAs) and lumbar lordosis angles (LLAs), by sex and by age.

\begin{tabular}{|c|c|c|c|}
\hline \multirow[t]{2}{*}{ Sex } & \multirow[t]{2}{*}{ Age (years) } & \multicolumn{2}{|c|}{ Sagittal angle of spine in natural standing position } \\
\hline & & $\operatorname{TKA}\left({ }^{\circ}\right)$ & $\operatorname{LLA}\left({ }^{\circ}\right)$ \\
\hline \multirow[t]{13}{*}{ Male } & 6 & 26.286(7.297) & $-32.500(9.504)$ \\
\hline & 7 & $34.583(9.053)$ & $-27.167(12.350)$ \\
\hline & 8 & $37.372(9.722)$ & $-24.488(9.480)$ \\
\hline & 9 & $36.797(8.944)$ & $-23.678(9.659)$ \\
\hline & 10 & $35.868(8.731)$ & $-24.015(9.770)$ \\
\hline & 11 & $38.341(7.886)$ & $-27.073(12.507)$ \\
\hline & 12 & $35.364(8.355)$ & $-25.697(9.554)$ \\
\hline & 13 & $31.383(5.968)$ & $-28.243(8.667)$ \\
\hline & 14 & $32.747(5.821)$ & $-26.670(8.352)$ \\
\hline & 15 & $34.273(5.147)$ & $-25.955(6.433)$ \\
\hline & 16 & $33.412(6.663)$ & $-25.618(9.912)$ \\
\hline & 17 & $31.500(6.658)$ & $-23.750(9.912)$ \\
\hline & 18 & $43.000(5.657)$ & $-27.500(3.536)$ \\
\hline \multirow[t]{11}{*}{ Female } & 6 & 27.906(7.731) & $-24.437(17.729)$ \\
\hline & 7 & 30.833(8.809) & $-23.738(12.057)$ \\
\hline & 8 & $34.463(11.227)$ & $-24.439(10.789)$ \\
\hline & 9 & $33.939(10.509)$ & $-23.653(8.430)$ \\
\hline & 10 & $37.527(10.059)$ & $-24.964(10.173)$ \\
\hline & 11 & $37.119(8.674)$ & $-25.619(10.138)$ \\
\hline & 12 & $32.333(8.222)$ & $-26.361(19.722)$ \\
\hline & 13 & $28.247(6.134)$ & $-26.194(13.193)$ \\
\hline & 14 & 28.393(5.995) & $-28.085(9.582)$ \\
\hline & 15 & $30.423(4.884)$ & $-26.462(7.495)$ \\
\hline & 16 & $29.377(6.968)$ & $-26.943(9.141)$ \\
\hline
\end{tabular}

Data represent mean (standard deviation). 


\begin{tabular}{|c|c|c|}
\hline 17 & $24.000(9.539)$ & $-34.667(7.234)$ \\
\hline 18 & $32.000(9.607)$ & $-36.000(4.950)$ \\
\hline
\end{tabular}

\section{Thoracic kyphosis angle}

As shown in Table 1, participant TKAs were significantly different for the three postures. In correlation analyses, we found that the TKAs for the reading/writing posture among 570 primary school and 568 secondary school participants were significantly and positively correlated with the TKAs of the natural standing posture $(p<0.01)$ with a significant linear relationship $\left(R^{2}=0.164 ; p<0.01\right)$ (Figs. 1 Figs. 2$)$. This result indicates that the read/writing position TKA is associated with the thoracic curve in participants when they stand naturally.

It is generally believed that the standard TKA should be $20^{\circ}$ to $40^{\circ}$, with angles greater than $40^{\circ}$ or less than $20^{\circ}$ considered excessive flexion and extension of the thoracic vertebrae $[16,17]$. Thus, we next examined the difference in the natural standing position TKA between participants with normal and abnormal $\left(<20^{\circ}\right.$ or $\left.>40^{\circ}\right)$ TKAs and found a significant difference $(p<0.01)$. When the TKA during reading/writing was in the standard reference range, then $83.1 \%$ of the participants had TKAs during standing in the normal reference range; conversely, when the TKA during standing was in the standard reference range, then $71.9 \%$ of the participants had TKAs during reading/writing in the normal reference range.

We also found a linear correlation between the TKAs for the postures during reading/writing and the upright sitting position $\left(R^{2}=0.380 ; p<0.01\right)$. This result indicated that secondary students with thoracic vertebrae that are too kyphotic in the reading/writing position have difficulty reaching the normal thoracic curvature angle when they sit upright.

\section{Lumbar lordosis angle}

Although the best LLA is unclear, maintaining lumbar lordosis is of substantial physiological significance [18], with many studies showing that abnormal lumbar curvature can lead to nonspecific low back pain $[9,19]$.

We found significant differences in LLAs among the three postures in primary and secondary school students. Most participants were able to maintain lumbar lordosis when standing naturally or sitting upright, but LLA was absent and lumbar kyphosis occurred in the reading/writing position (Table 1).

We stratified the LLAs for the three postures according to whether lumbar lordosis could be maintained [20]. Nearly all students (99.1\%) who could maintain lumbar lordosis in the seated reading/writing position could also maintain it in the other two postures. By contrast, there was a significant difference in the natural standing LLA for students who maintained vs. those who lost LLA during seated 
reading/writing. These results suggested that there was a significant interaction between the seated reading/writing position and the natural standing LLA such that students who maintained standard reference LLAs in a seated reading/writing position had a healthy lumbar curvature when standing naturally, and vice versa.

There was also a significant correlation between thoracic and lumbar angles in different postures. Among all participants, $93.7 \%$ of the 774 students who were able to maintain the standard reference range for the TKA in seated reading/writing position could also maintain normal lumbar lordosis while sitting upright, indicating the benefit of maintaining lumbar lordosis.

\section{Association between thoracic curvature and vital capacity}

Studies have shown that body posture affects respiratory function and the range of motion of the chest and diaphragm [21].

A scatter plot of the association between TKA during natural standing and vital capacity (Fig. 3), shows an obvious inverted $U$ shape. Using independent-samples $t$ test analyses, we found that the vital capacity of students with a normal TKA (defined as between $20^{\circ}$ and $40^{\circ}$ ) was significantly higher than that of students with a greater or lesser TKA $(p<0.01)$.

\section{Body posture and sagittal plane angle of the spine}

Body posture, as indicated by the pelvic inclination and neck inclination of the natural standing posture, was measured using a PA200 Station Posture Assessment System. We found a significant linear association between the inclination angle and the pelvic TKA of the seated reading/writing position in primary school participants $(p<0.01)$ (Fig. 4$)$.

\section{Height and sagittal plane angle of the spine}

We found significant correlations between the height of primary and of secondary school students and their seated reading/writing TKAs and LLA $(p<0.05)$. To further assess the effect of height on the shape of the spine in these students, we analyzed their mean TKAs and LLAs by 5 -cm increments in height. Figures 5 and 6 show significant positive correlations between TKA and height and between LLA and height in secondary school students. Lumbar curvature began to change from lordosis to kyphosis once individuals were taller than $170 \mathrm{~cm}$, suggesting that the desks and chairs they were using use were no longer suited to their height. The thoracic curvature also increased linearly with height in primary school children, but their thoracic kyphosis angles were within the normal reference range. Although there was no linear association between lumbar curvature and height in primary school children, almost all the students had lumbar kyphosis when reading and writing at their desks.

\section{Discussion}


Humans have evolved over tens of thousands of years to maintain an upright posture, causing some parts of the body to be under a great deal of pressure while standing. To relieve vertical pressure, the human spine has evolved four physiological bends that, in addition to buffering concussion, also support the movement of the torso in the sagittal and coronal planes [1]. The movement of the torso in the sagittal plane is mainly undertaken by the neck, chest, and waist. When in the natural standing position, the muscles used to maintain the physiological curves in the spine are in the most relaxed state. The physiological curvature of the spine is also closest to the natural state when the body is very young, but the spine is prone to pathological deviations under prolonged improper posture [2, 3, 22].

Our study found that comparing natural standing to an upright sitting posture, the physiological curvature of the spine is greatly adjusted in the sitting position, resulting in a tendency for increase in thoracic curvature, and loss of lumbar kyphosis. The thoracic curvature, a more stable segment of the spine, has a smaller angle of motion and thus is easier to maintain, whereas the lumbar curvature, a more flexible segment, has a larger range of motion [2]. With increasing sagittal changes of the spine, angle changes and loss of lordosis bring a greater load to the lumbar spine. Previous imaging evidence has shown that when a person sits in a relaxed posture on a stool, the entire lumbar lordosis below the lumbar L1-L2 level becomes more kyphotic, and lumbar lordosis at L4-L5 and the lumbosacral joint L5-S1 levels decreases significantly. These results indicate the relaxation of the sacrospinalis and multifidus muscle of the waist [23], which indicates that the vertebral body has a high compression load in this position.

Our results showed that the standing pelvic angle was significantly correlated with TKA, and the neck inclination angle was significantly correlated with LLA. Because the range of motion of the sacroiliac joint is very small, the pelvic inclination measured by the PA200 system was similar to the sacral tilt angle of the participants[24].The human pelvis gradually tilts forward from sitting to standing [25, 26],thus, the sitting posture has a lower sacral inclination angle than the standing posture. We speculate that the decrease of the pelvic oblique angle during seated reading/writing leads to decreased lumbar lordosis and even to lumbar kyphosis. When lumbar support is reduced or absent, thoracic kyphosis will increase accordingly [27]. Students seated improperly at a desk for a long time may experience increased kyphosis, and TKA increases substantially, exceeding the normal kyphosis angle range of $20^{\circ}$ to $40^{\circ}$. At the same time, to maintain the distance between the eyes and the reading/writing material, the cervical vertebra in the sagittal plane adapts as a whole as a compensatory mechanism for maintaining horizontal gaze.

Most secondary school students who sit at chair/desk combinations that are too small for their height are unable to maintain a good sitting posture. When they continue in this position in order to see the word, high-intensity pressure will inactivate the local spinal stabilizing muscles that resist fatigue and increase tension on the connective tissue. The shape and posture of the spine will become set, even when the student is standing naturally. In addition, excessive anterior traction of the neck increases the load of the muscles in the shoulder and neck and induces upper cross syndrome [28,29], while the loss of lumbar lordosis is the direct cause of low back pain [27, 30]. Excessive changes in thoracic spine curvature can also affect pulmonary ventilation (Fig. 3). Culham et al. compared the vital capacity of normal women 
and women with thoracic kyphosis caused by osteoporosis and found that vital capacity and rib mobility were significantly impaired in women with thoracic kyphosis[31]. Therefore, a normal chest curve is essential to the volume of the chest and the ventilatory function of the lungs [21, 32]. Our conclusions confirm and extend those of that study. The effect of the chest curvature on vital capacity was substantial, even when no pathological thoracic kyphosis was present, vital capacity was significantly changed with TKA. By contrast, we found that participants who were able to maintain TKA and LLA within standard references ranges while seated reading/writing at their desks had better spine shape while standing. This highlights the importance of a good sitting position.

The analysis of height and spine morphology we performed reflects the hidden spinal issue among secondary school students: the taller the students, the more likely they are to stoop and hunch. When children enter puberty rapidly, some may grow tall faster than their peers, leading to a greater difference in their heights. Thus, desks and chairs that are only one size are not suitable for the various heights of students. Taller students must use more body flexion in a desk that is too small to maintain the correct distance from a textbook to clearly see it. This flexion is accomplished not only by increasing thoracic curvature but also by reducing lumbar lordosis. This is reflected in the participants' mean TKAs and LLAs (Table 1). However, most primary and secondary schools in China do not have the ability to purchase desks and chairs based on the various heights of students.

If the pelvis is rotated forward during development while sitting, sciatic support is reduced, lumbar support is strengthened to obtain neutral lordosis of the lumbar spine, and the thoracic cavity is relaxed. These changes cause synergistic activation of the superficial lumbar multifidus muscles and internal oblique muscles, resulting in local stabilization of the lumbosacral region without a high compressive load $[19,23]$. However, most primary and secondary schools in China have desks and chairs that are not equipped with supporting cushions for the lumbar vertebrae and thus do not provide stable support for students' lumbar curvature.

The majority of studies on the morphology of the spine have focused on the coronal plane, with few studies assessing the sagittal plane of the spine in children and teenagers [33]. As teenagers spend increasingly more time sitting, the deformation of their spine in the sagittal plane is becoming a more common and noteworthy problem. Our results remind teachers of the importance of requiring students to maintain good sitting posture at all times. Reducing students' sedentary time or providing students with desks and chairs suitable for their height or that maintain their sacral oblique angle may have a positive effect on improving the sitting posture among teenagers in China.

\section{Conclusion}

When primary and secondary school students reading/writing, the thoracic kyphosis angle and the lumbar lordosis angle decreased significantly and even kyphosis appeared. The sitting posture is highly correlated with the sagittal angle of the spine of standing and upright position. Poor sitting posture will change subjects' spine shape. We speculate that this is the result of the body's re-adaptation which was 
caused by long-term deviation of the spine shape. When students use desks and chairs with the same height, the height of the students becomes an important factor affecting the shape of teenagers' sitting spines. Taller students' spines need more flexion and are more prone to imbalance. Therefore, the use of desks and chairs with appropriate height or lumbar support may be conducive to maintain proper spinal shape and alignment.

\section{Abbreviations}

TKA: thoracic spine kyphotic angle; LLA: lumbar lordosis angle; SD: Standard

deviation; CR: Coefficient of repeatability

\section{Declarations}

\section{Ethical approval statement}

The whole process of this study was carried out under the requirements of the Helsinki Declaration. The reporting of this study followed the Strengthening the Reporting of Observational Studies in Epidemiology (STROBE) reporting guideline for observational studies.

This study was conducted as approved by the institutional review board of Tianjin University of Sport, Tianjin, China (approval number TJUS2017015). All participants and their guardians understood the purpose and method of this study.

\section{Informed consent to participate}

Informed consent was obtained from the participant's parents if the participant was under 16 years of age, otherwise informed consent was obtained from the participants themselves.

\section{Consent for publication}

Not applicable.

\section{Availability of data and materials}

The datasets used and/or analysed during the current study are available from the corresponding author on reasonable request.

\section{Competing interests}

The authors declare that they have no competing interests.

\section{Funding}


This research received no specific grant from any funding agency in the public, commercial or not-forprofit sectors.

\section{Authors' contributions}

LCQ did the study design, analyses and drafted the initial manuscript. ZYQ and HX conducted data collection and preliminary analyses. All authors (LCQ, ZYQ, YZH, HX, WL and LX) contributed to analysis and interpretation of results, conclusions and dissemination. $\mathrm{WL}$ and $\mathrm{LX}$ was the co-corresponding author. All authors read and approved the final manuscript.

\section{Acknowledgements}

Not applicable

\section{References}

1. Morris JM. Biomechanics of the spine. Archives of surgery (Chicago, III : 1960). 1973;107(3):41823.

2. Adams MA, Dolan P. Spine biomechanics. Journal of biomechanics. 2005;38(10):1972-83.

3. Bergmark A. Stability of the lumbar spine. A study in mechanical engineering. Acta Orthop Scand Suppl. 2009;60(s230):1-54.

4. Miele VJ, Panjabi MM, Benzel EC. Anatomy and biomechanics of the spinal column and cord. Handbook of clinical neurology. 2012;109:31-43.

5. Diebo BG, Varghese JJ, Lafage R, Schwab FJ, Lafage V. Sagittal alignment of the spine: What do you need to know? Clinical neurology and neurosurgery. 2015;139:295-301.

6. Joao Paulo C, Peter OS, Angus B, Avi B, David ON, Orjan T, et al. The influence of different sitting postures on head/neck posture and muscle activity. Man Ther. 2010;15(1):54-60.

7. Czupryna K, Nowotny-Czupryna O, Nowotny J. Neuropathological aspects of conservative treatment of scoliosis. A theoretical view point. Ortopedia Traumatologia Rehabilitacja. 2012;14(2):103.

8. Szczygieł E, Zielonka K, Mętel S, Golec J. Musculo-skeletal and pulmonary effects of sitting position - A systematic review. Annals of Agricultural \& Environmental Medicine Aaem. 2017;24(1).

9. Balagué $F$, ., Nordin M, ., Skovron ML, Dutoit G, ., Yee A, ., Waldburger M, . Non-specific low-back pain among schoolchildren: a field survey with analysis of some associated factors. Journal of Spinal Disorders. 1994;7(5):374-9.

10. Calvo-Munoz, Inmaculada, Gomez-Conesa, Antonia, Sanchez-Meca, Julio. Prevalence of low back pain in children and adolescents: a meta-analysis. Bmc Pediatrics. 2013;13(1):14- 
11. Miyake A, Kou I, Takahashi Y, Johnson TA, Ogura Y, Dai J, et al. Identification of a Susceptibility Locus for Severe Adolescent Idiopathic Scoliosis on Chromosome 17q24.3. Plos One. 2013;8(9):130-.

12. Zhou S, Sun Z, Li W, Wang W, Su T, Du C, et al. The standing and sitting sagittal spinopelvic alignment of Chinese young and elderly population: does age influence the differences between the two positions? European spine journal : official publication of the European Spine Society, the European Spinal Deformity Society, and the European Section of the Cervical Spine Research Society. 2019.

13. Aggarwal N, Anand T, Kishore J, Ingle GK. Low back pain and associated risk factors among undergraduate students of a medical college in Delhi. Education for Health. 2014;26(2):103.

14. Agar G. Assessing kyphosis with SpineScan: another attempt to reduce our dependence on radiography. Spine Journal.13(8):926-31.

15. Landis JR, Koch GG. The Measurement of Observer Agreement for Categorical Data. Biometrics.33(1):159-74.

16. Tribus CB. Scheuermann's kyphosis in adolescents and adults: diagnosis and management. J Am Acad Orthop Surg. 1998;6(1):36-43.

17. Lowe TG. Scheuermann's kyphosis. Neurosurgery Clinics of North America. 2007;18(2):305-15.

18. Claus AP, Hides JA, Moseley GL, Hodges PW. Is 'ideal' sitting posture real?: Measurement of spinal curves in four sitting postures. Manual Therapy. 2009;14(4):404-8.

19. Makhsous M, Lin F, Bankard J, Hendrix RW, Hepler M, Press J. Biomechanical effects of sitting with adjustable ischial and lumbar support on occupational low back pain: evaluation of sitting load and back muscle activity. BMC musculoskeletal disorders. 2009;10:17.

20. Xia W, Fu H, Zhu Z, Liu C, Wang K, Xu S, et al. Association between back muscle degeneration and spinal-pelvic parameters in patients with degenerative spinal kyphosis. BMC musculoskeletal disorders. 2019;20(1):454.

21. Kaneko H, Horie J. Breathing Movements of the Chest and Abdominal Wall in Healthy Subjects. Respir Care. 2012;57(9):1442-51.

22. O'Sullivan PB, Wim D, Burnett AF, Farrell GT, Evonne J, Naylor CS, et al. Effect of different upright sitting postures on spinal-pelvic curvature and trunk muscle activation in a pain-free population. Spine. 2006;31(19):707-12.

23. O'Sullivan P, Dankaerts W, Burnett A, Chen D, Booth R, Carlsen C, et al. Evaluation of the Flexion Relaxation Phenomenon of the Trunk Muscles in Sitting. Spine.31(17):2009-16. 
24. Legaye J, Duval-Beaupère G, Hecquet J, Marty C. Pelvic incidence: a fundamental pelvic parameter for three-dimensional regulation of spinal sagittal curves. European spine journal : official publication of the European Spine Society, the European Spinal Deformity Society, and the European Section of the Cervical Spine Research Society. 1998;7(2):99-103.

25. De Carvalho DE, Soave D, Ross K, Callaghan JP. Lumbar spine and pelvic posture between standing and sitting: a radiologic investigation including reliability and repeatability of the lumbar lordosis measure. Journal of manipulative and physiological therapeutics. 2010;33(1):48-55.

26. Jun Seok B, Jee-Soo J, Sang-Ho L, Uk KJ. A comparison study on the change in lumbar lordosis when standing, sitting on a chair, and sitting on the floor in normal individuals. Journal of Korean Neurosurgical Society. 2012;51(1):20-3.

27. Barrey C, Pinheiro-Franco JL, Le-Huec JC, Perrin G, Roussouly P. Compensatory Mechanisms Contributing to the Maintenance of Sagittal Balance in Degenerative Diseases of the Lumbar Spine2016.

28. Grob D, Frauenfelder H, Mannion AF. The association between cervical spine curvature and neck pain. European Spine Journal. 2007;16(5):669-78.

29. Fan Y, Wang J, Cai M, Wang X, Xia L. The correlation between postoperative cervical sagittal alignment and spine sagittal alignment in adolescent idiopathic scoliosis: a meta-analysis. World neurosurgery. 2019.

30. Glassman SD, Bridwell K, Dimar JR, Horton W, Berven S, Schwab F. The impact of positive sagittal balance in adult spinal deformity. Spine (Phila Pa 1976). 2005;30(18):2024-9.

31. Culham EG, Jimenez HA, King CE. Thoracic kyphosis, rib mobility, and lung volumes in normal women and women with osteoporosis. Spine (Phila Pa 1976). 1994;19(11):1250-5.

32. Lee LJ, Chang AT, Coppieters MW, Hodges PW. Changes in sitting posture induce multiplanar changes in chest wall shape and motion with breathing. Respiratory physiology \& neurobiology. 2010;170(3):236-45.

33. Gonzalez-Iglesias J, Gutierrez-Vega-Mdel R. Thoracic spine manipulation for the management of patients with neck pain: a randomized clinical trial. Journal of Orthopaedic \& Sports Physical Therapy. 2009;39(1):20.

34. State General Administration of Quality Supervision, Inspection and Quarantine. GB/T3976-2014. Functional Dimensions and Technical Requirements of School Desks and chairs. Beijing: China Standard Press, 2014:9. 
Figures
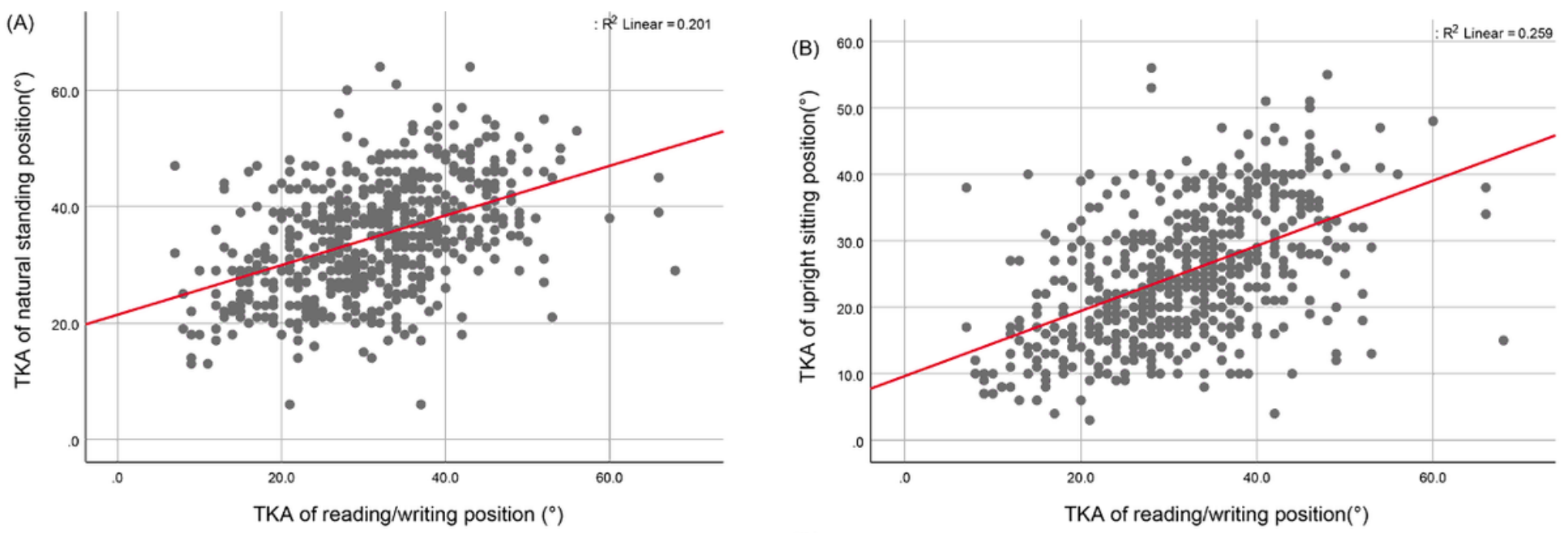

(C)
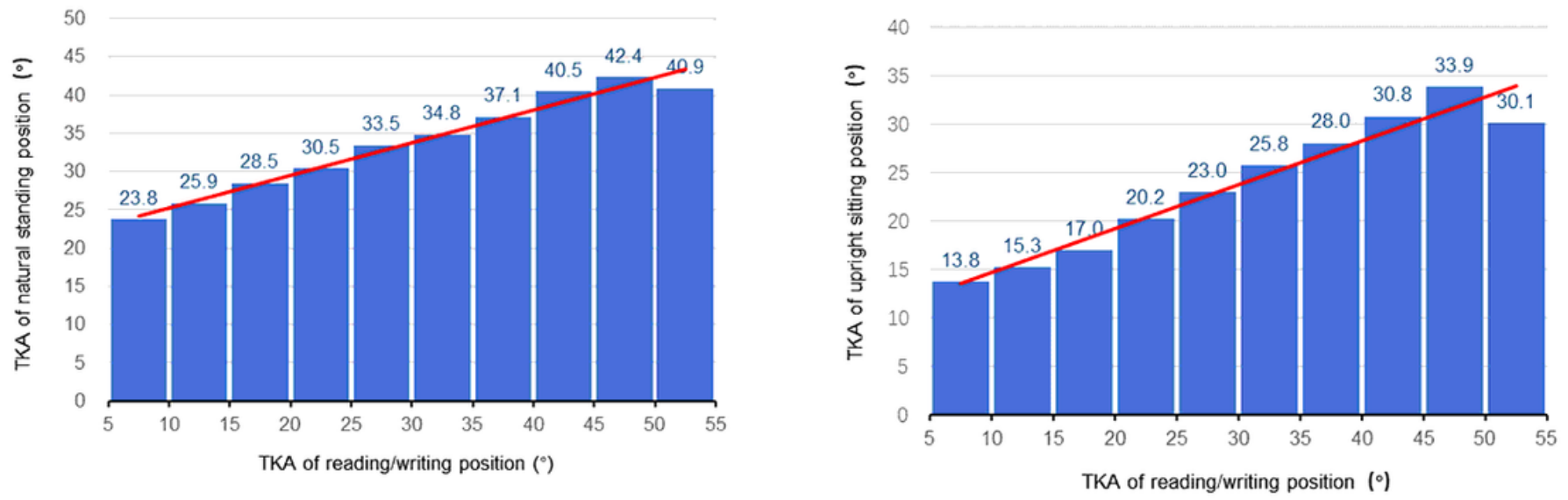

\section{Figure 1}

Correlations and histograms of thoracic kyphosis angles (TKAs) in the seated reading/writing position vs. the natural standing position or an upright sitting position in primary school children. 


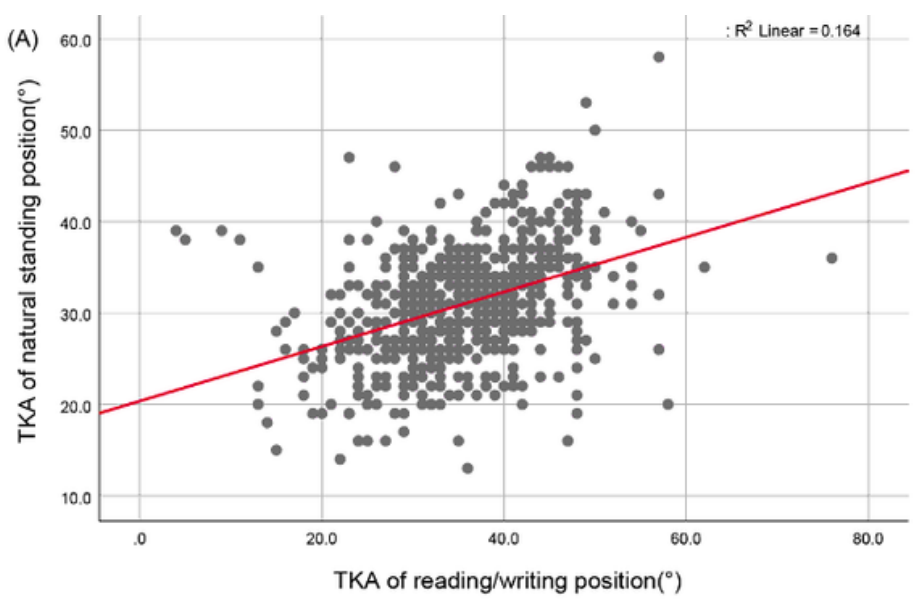

(C)

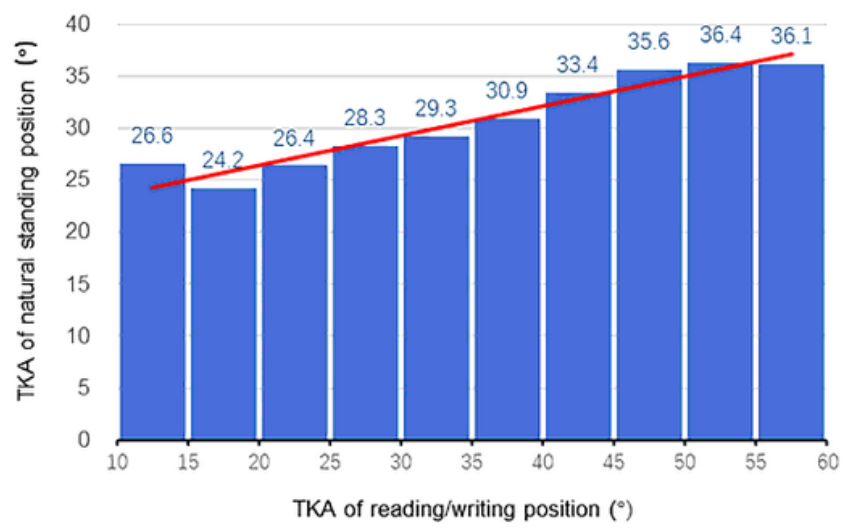

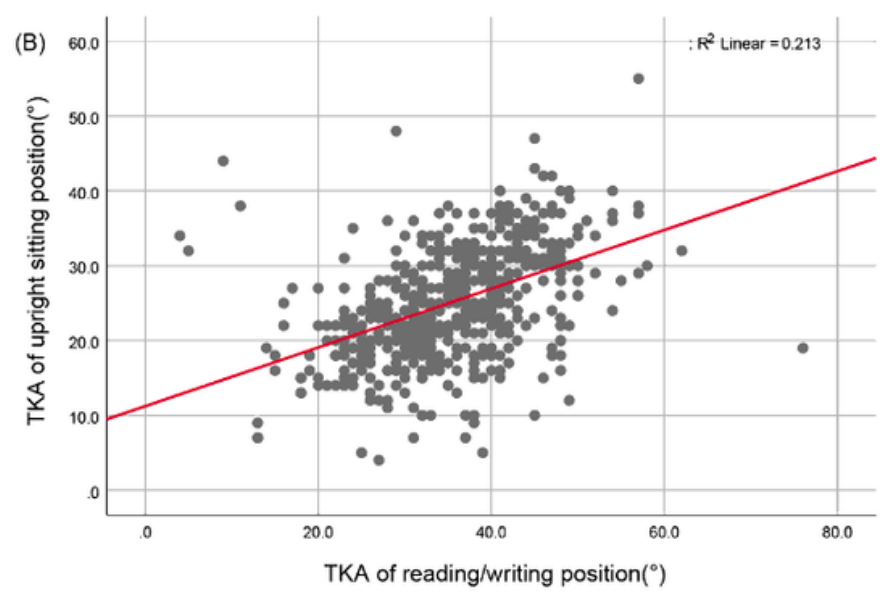

(D)

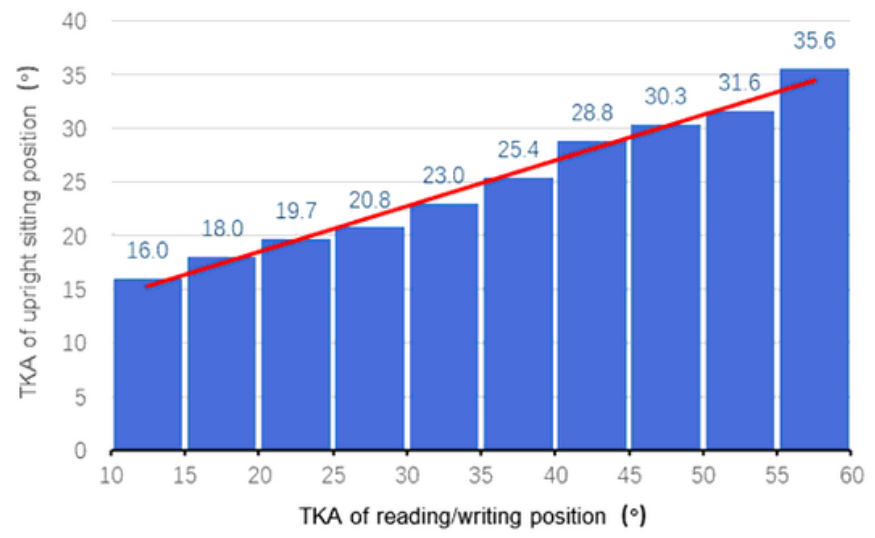

Figure 2

Correlations and histograms of thoracic kyphosis angles (TKAs) during reading/writing vs. a natural standing position or vs. an upright sitting position in secondary school students. 


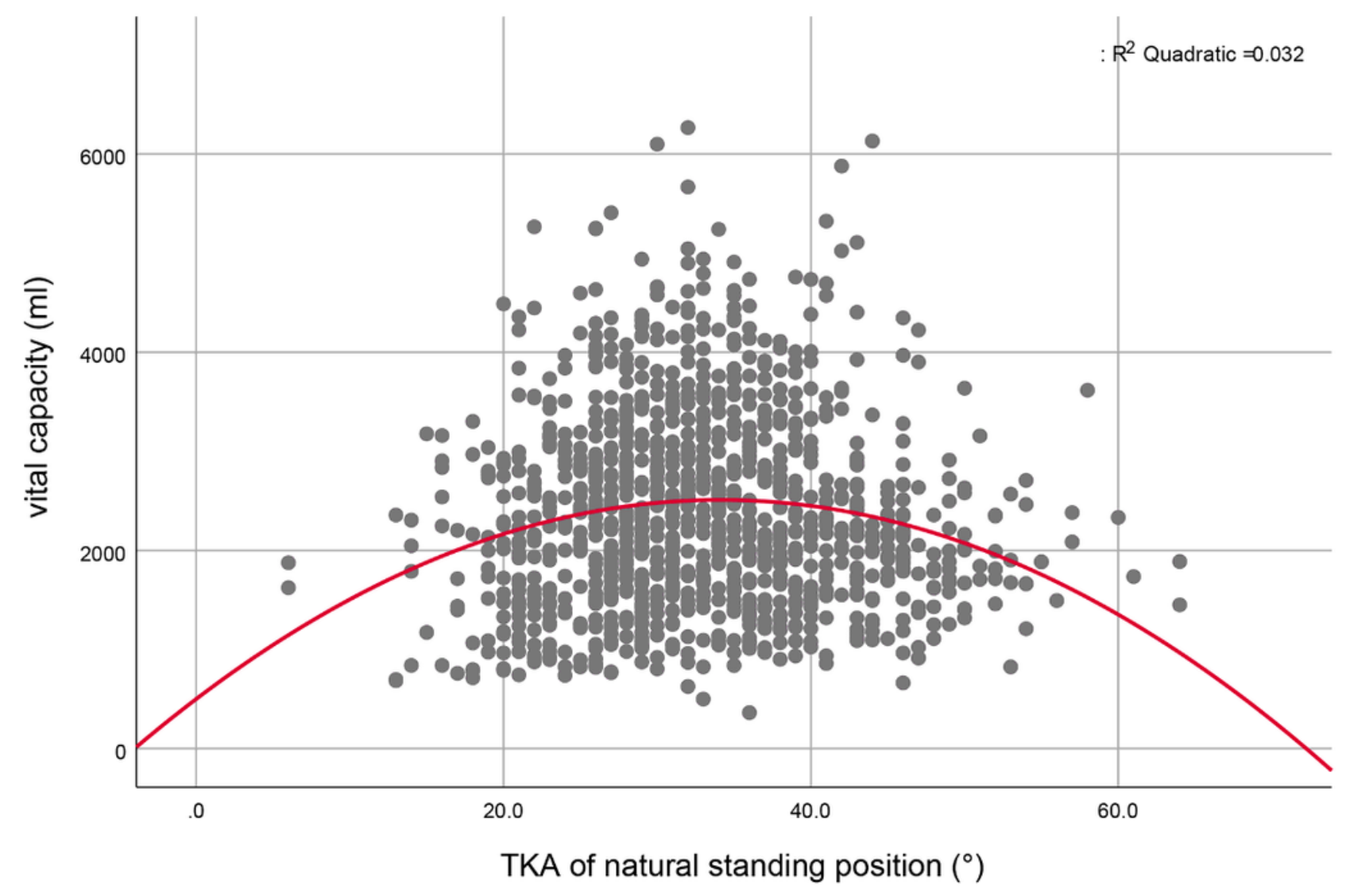

Figure 3

Scatter diagram of the association between the natural standing position thoracic kyphosis angle (TKA) and vital capacity. 


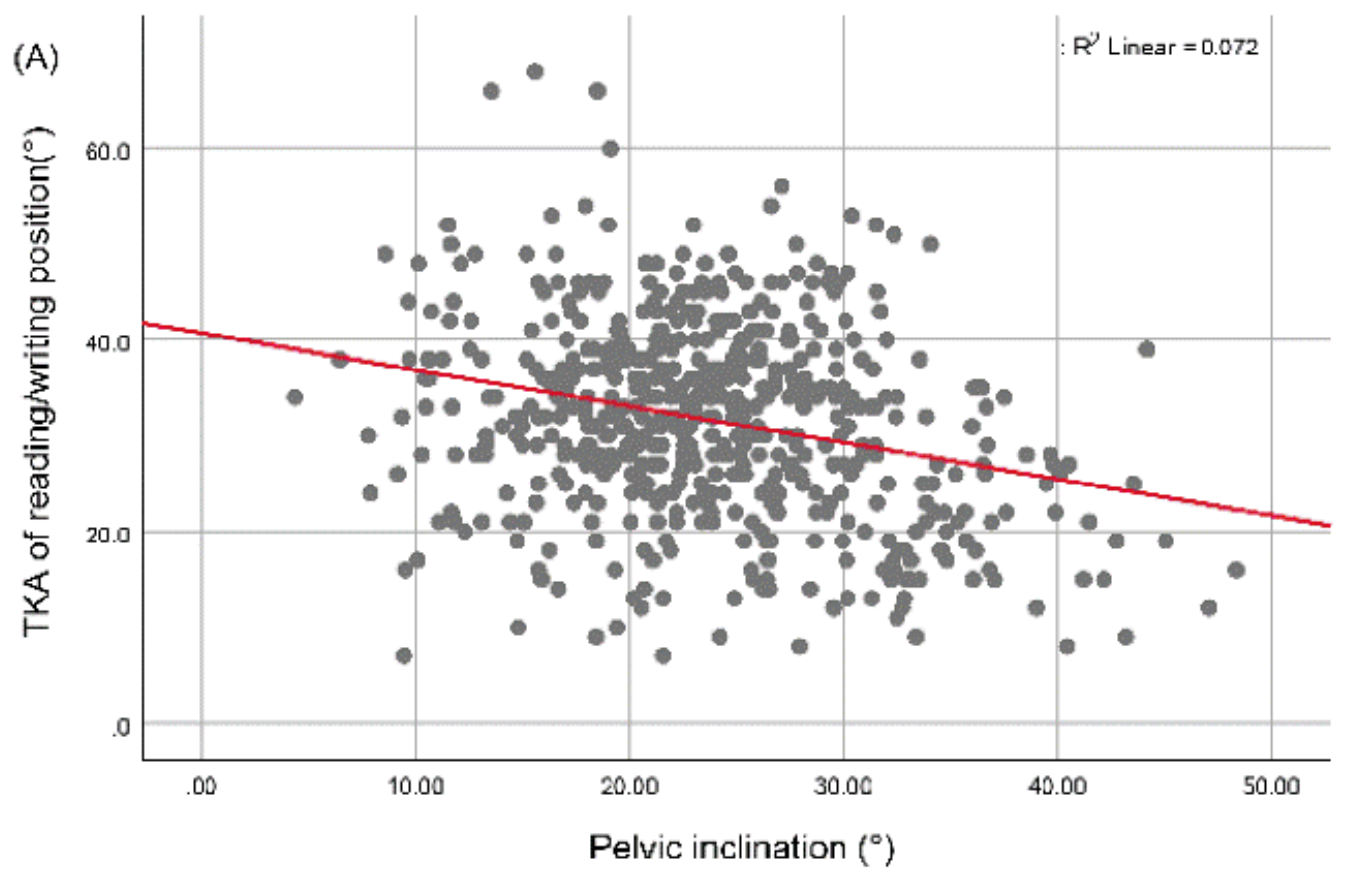

(B) 40

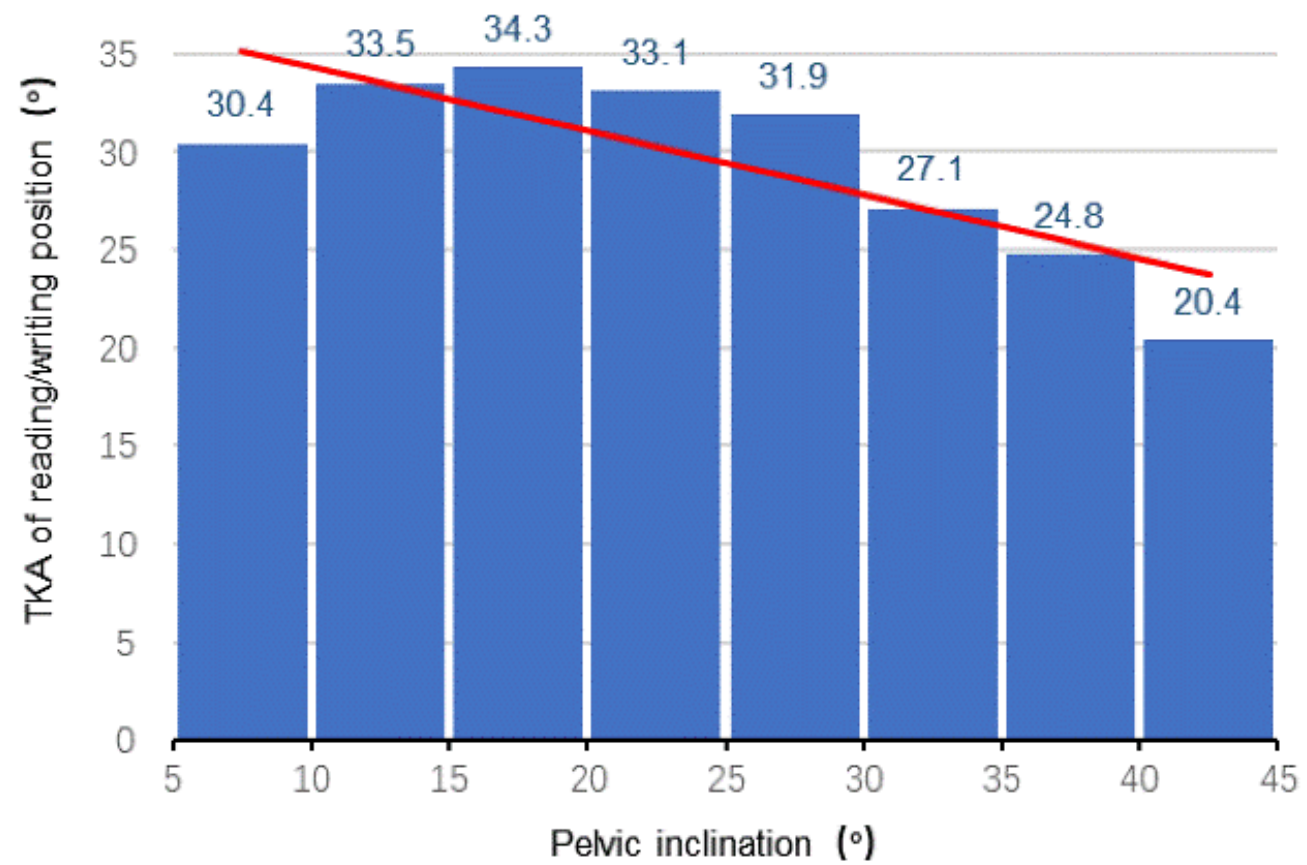

Figure 4

Scatter plot and histogram showing the association between pelvic inclination and seated reading/writing thoracic kyphosis angle (TKA) in primary school children. 

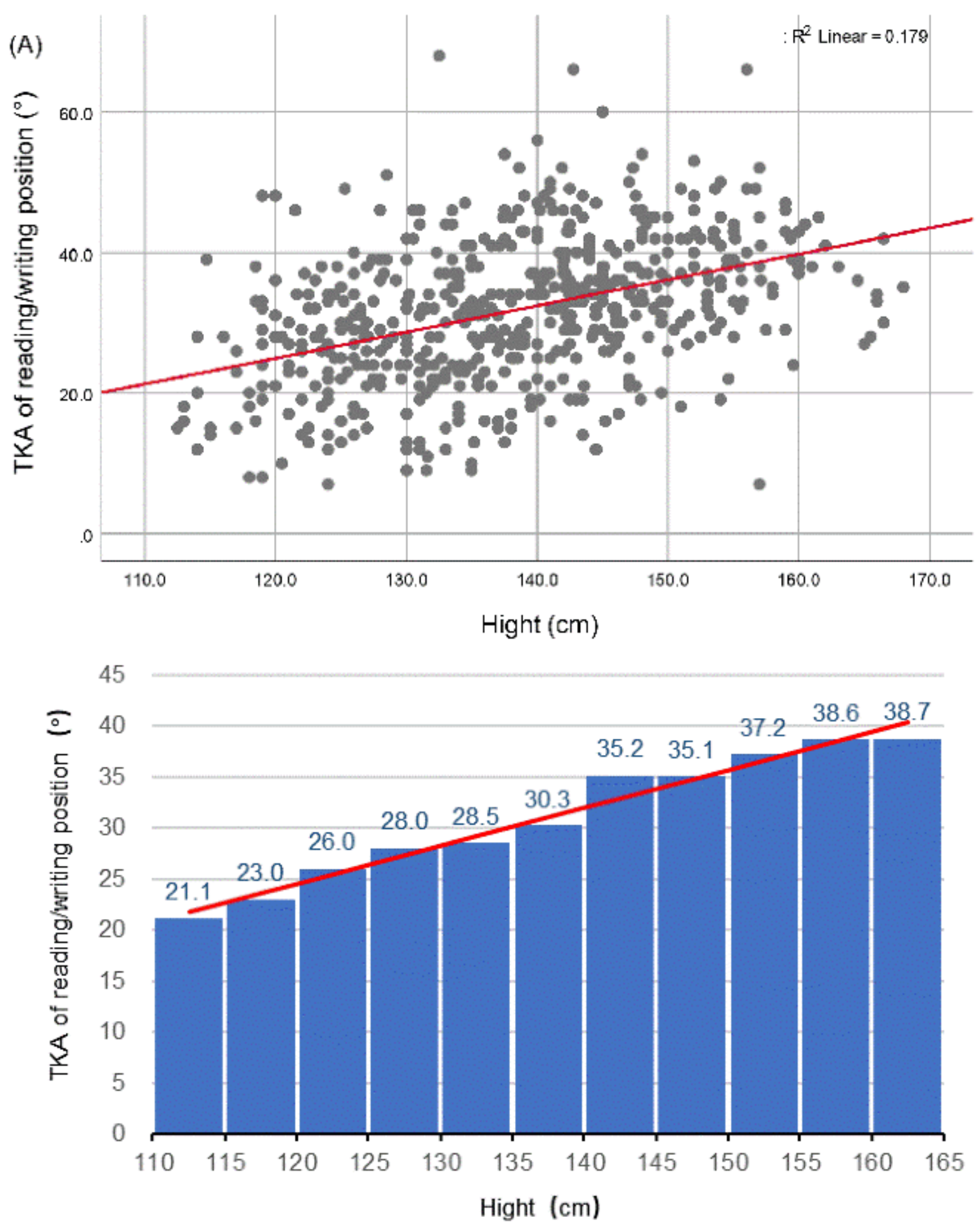

Figure 5

Scatter plot and histogram showing the association between and seated reading/writing thoracic kyphosis angle (TKA) and height in primary school children. 

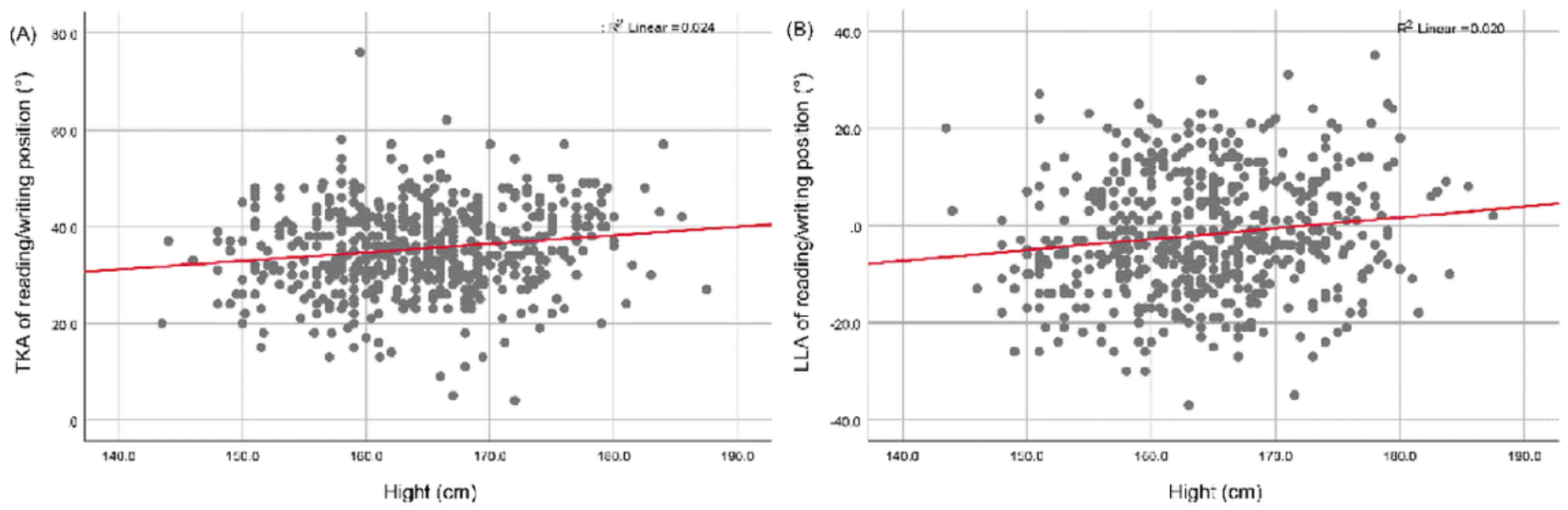

(C)

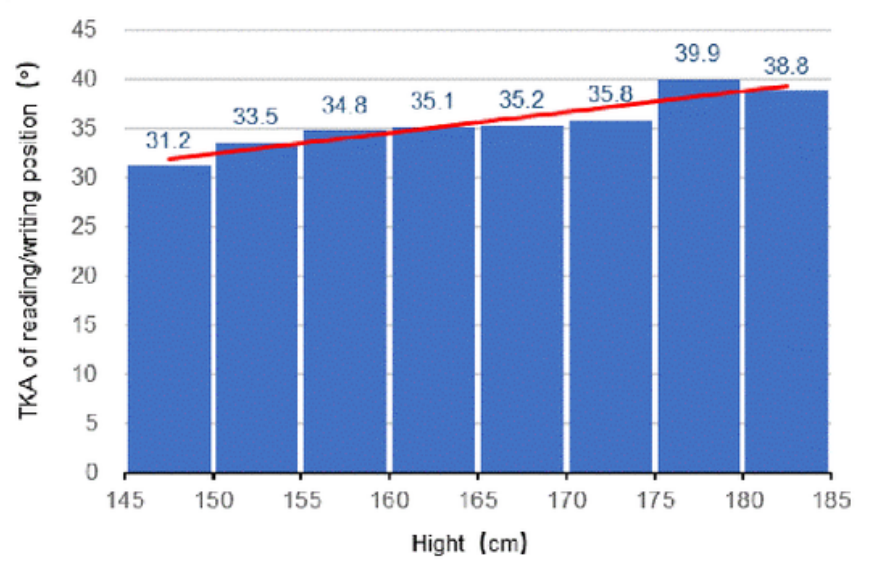

(D)

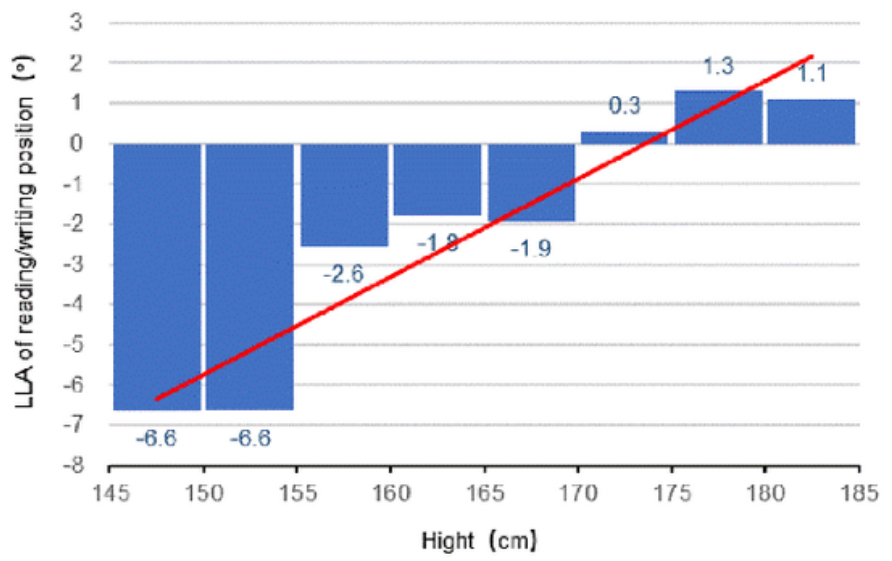

Figure 6

Scatter plots and histograms showing the associations of seated reading/writing thoracic kyphosis angle (TKA) and lumbar lordosis angle (LLA) with height in secondary school students. 